\title{
Colon capsule endoscopy for detection of polyps and cancers: a step closer to non-invasive colon screening?
}

\author{
${ }^{1}$ A Koulaouzidis, ${ }^{2} \mathrm{JN}$ Plevris \\ ${ }^{1}$ Associate Specialist; ${ }^{2}$ Consultant and Reader in Gastroenterology, Centre for Liver \& Digestive Disorders, Royal Infirmary of Edinburgh, \\ Edinburgh, UK
}

TITLE Capsule Endoscopy versus Colonoscopy for the Detection of Polyps and Cancer

AUTHORS Van Gossum A, Munoz-Navas M, Fernandez-Urien I, et al.

JOURNAL N Engl J Med 2009; 361:264-70 doi:10.1056/NEJMoa0806347

DECLARATION OF INTERESTS No conflicts of interest declared.

\author{
Correspondence to J Plevris, \\ Centre for Liver \& Digestive \\ Disorders, Royal Infirmary of \\ Edinburgh, 5 I Little France Crescent, \\ Edinburgh EHI6 4SA, UK
}

tel. +44 (0) I3। 242 I63I

e-mail j.plevris@ed.ac.uk

\section{SUMMARY}

Most colorectal screening programmes use colonoscopy as the gold standard for colon examination, although several factors including invasiveness, inconvenience, discomfort, potential complications or simply embarrassment significantly limits its take-up by the asymptomatic general population.' Wireless capsule endoscopy has already been established as the state-ofthe-art non-invasive diagnostic imaging modality for the small bowel, therefore a modified capsule for investigation of the colon appears as an attractive option.

In this New England Journal of Medicine article, ${ }^{2}$ the results of a prospective multi-centre study comparing conventional colonoscopy with colon capsule endoscopy (CCE) were reported. The study was conducted in eight European centres and recruited 332 patients. All subjects belonged to the high-risk group, as they had either known $(n=112)$ or suspected $(n=208)$ colonic disease and were scheduled to undergo further colonic investigations. They ingested the double-headed first generation of PillCam $®$ COLON capsule $(31 \times 1 \mathrm{l} \mathrm{mm})$, prior to having a conventional colonoscopy. They all received a vigorous (adapted for the needs of colon capsule) preparation for large bowel cleansing. Colon capsule endoscopy and colonoscopy findings were reported independently. The investigators computed the sensitivity and specificity of the CCE in detecting number of polyps, polyps $>1 \mathrm{~cm}$ in size and cancers.

The sensitivity and specificity of the CCE for the detection of polyps $>6 \mathrm{~mm}$ in size were $64 \%$ and $84 \%$ respectively, while for polyps $>1 \mathrm{~cm}$ those values were $73 \%$ and $79 \%$ respectively.Additionally, of the 19 colon cancers detected by conventional colonoscopy, only 14 were also detected by the CCE. A significant number of patients (7.9\%) reported adverse effects, mostly to do with the intensity of the bowel preparation. Finally, the negative predictive value of the colon capsule was high at levels of $90 \%$.

\section{OPINION}

Since its introduction in clinical practice in 200I, capsule endoscopy found its niche in the investigation of the small bowel. More accessible parts of the gastrointestinal tract (oesophagus and colon) have since been targeted by double-headed devices produced by Given Imaging Ltd. However, results of recent meta-analyses are not in favour of oesophageal capsule endoscopy, while for CCE, one of the most important impediments remains the lack of any therapeutic capacity. It therefore introduces a different philosophy among the practicing colonoscopists who have been taught to look for and remove most polyps.

Indeed, even when excluding polyps $<5 \mathrm{~mm}$ in size, van Gossum et al. ${ }^{2}$ showed that the sensitivity and specificity for adenoma detection rate was rather disappointing. Furthermore, five colon cancers were missed by capsules but were picked up by colonoscopy. This is of concern in the clinical context of the study.

Capsule examination is generally appreciated for its non-invasive nature. Admittedly though, one of the major practical challenges in performing CCE (and in contrast to small bowel capsule examination), is the vigorous bowel preparation which at present involves taking four litres of a cleansing laxative, in addition to some boosters during the test. It is not difficult to guess that this rather demanding bowel cleansing regimen, albeit necessary for polyp detection, can only be achieved safely either in younger, fit and highly motivated patients or those hospitalised for close observation. Not surprisingly, the reported complications in this study were mostly bowel-prep related. ${ }^{2}$ This factor alone could act as a significant deterrent in achieving adherence with a CCE-based screening platform, a factor so important for the acceptability of any screening method. 
A further concern is the ability of the CCE to produce images that reliably inspect the majority of the bowel mucosa. Despite the extended battery time, the adaptive frame rate with automatic light control and the presence of two imager domes for simultaneous forward and retrograde viewing, the current design cannot prevent the capsule tumbling around in the wide colon lumen thus not maintaining a stable luminal view, resulting in potentially missed pathology.

An alternative and increasingly acceptable colorectal screening investigation that directly competes with both conventional colonoscopy and CCE is computed tomography (CT) colonography. The latest generation of CT scanners provide excellent resolution and ability to reliably detect clinically significant polyps of $6 \mathrm{~mm}$ or more in addition to imaging the rest of the intraabdominal organs. ${ }^{3,4}$ Furthermore, the refinement of the technique of minimal preparation CT colonography overcomes the challenges of vigorous colon preparation and this test becomes particularly suitable for frail and elderly patients. ${ }^{5}$

Despite the above problems and competition with 'attractive' alternative techniques, ${ }^{3}$ CCE is likely to become an option for colorectal screening in the future as the technology improves and less vigorous bowel preparations are successfully being employed. New prototype colon capsule systems have already been designed to improve luminal views; in a recent article, a new colon capsule system with a semi-permeable rear compartment able to absorb fluid so as to change the capsule shape to a more hydrodynamic one has been reported, thus improving stability, which translates into more complete colon luminal views. ${ }^{6}$

At present, CCE may be reserved for some selected and highly motivated groups of patients who can tolerate the vigorous bowel preparation and for whom it is desirable to get colon mucosal views in the least invasive way. Such groups include patients with coagulation disorders (such as haemophilia or von Willebrand disease) who often present with positive for blood stool samples on colorectal screening and would normally require expensive blood products infused to correct coagulation prior to conventional colonoscopy.

\section{CONCLUSION}

Conventional colonoscopy remains the cornerstone of a successful bowel screening programme either as a primary investigation or following a positive for blood stool sample, not only as a diagnostic but also as a therapeutic tool. Computed tomography colonography closely competes with conventional colonoscopy in terms of diagnosis as it is less invasive but lacks the therapeutic component. Colon capsule endoscopy has not as yet reached its full potential, but may be of value to selected groups such as patients with coagulation disorders.

\section{REFERENCES}

I Steele RJ, McClements PL, Libby G et al. Results from the first three rounds of the Scottish demonstration pilot of FOBT screening for colorectal cancer. Gut. 2009; 58:530-5. doi:10.1136/ gut.2008.162883

2 Van Gossum A, Munoz-Navas M, Fernandez-Urien I et al. Capsule endoscopy versus colonoscopy for the detection of polyps and cancer. N Engl J Med. 2009 16; 361:264-70. doi:10.1056/ NEJMoa0806347

3 Pickhardt PJ, Choi JR, Hwang I et al. Computed tomographic virtual colonoscopy to screen for colorectal neoplasia in asymptomatic adults. N Engl J Med. 2003; 349:2191-200. doi:10.1056/NEJMoa031618

4 Johnson CD, Chen MH, Toledano AY et al. Accuracy of CT colonography for detection of large adenomas and cancers. N Engl J Med. 2008; 359: I207-17. doi: I0.1056/NEJMoa0800996

5 Jensch S, de Vries AH, Peringa J et al. CT colonography with limited bowel preparation: performance characteristics in an increasedrisk population. Radiology. 2008; 247:122-32. doi:10.1/48/ radiol.247I070439

6 Filip D, Yadid-Pecht O, Andrews CN et al. Improved Video Quality and Reliability With Self-Stabilizing Colon Capsule Endoscopy: Pilot Study in Acute Canine Models. Gastroenterology 201 I; I40: SII9. 